\title{
Analytic models for a spherical satellite charging in sunlight at any spin rate
}

\author{
M. Tautz ${ }^{1}$ and S. T. Lai ${ }^{2}$ \\ ${ }^{1}$ AER/Radex Inc., Bedford, MA 01730, USA \\ ${ }^{2}$ Space Vehicles Directorate, AFRL/ VSBXT, Air Force Research Laboratory, Hanscom AFB, MA, 01731-3010, USA
}

Received: 20 October 2005 - Revised: 1 August 2006 - Accepted: 24 August 2006 - Published: 20 October 2006

\begin{abstract}
We present analytic models for the steady state potential distributions surrounding a spinning, dielectriccoated, spherical spacecraft charging in sunlight. The sun direction is assumed to lie in the satellite bellyband plane, perpendicular to the spin axes. The models are based on a multipole expansion of Laplacian potentials external to the spacecraft surface. The combination of monopole potentials along with the dipole or quadrupole contributions produce potential barriers which form at the satellite surface. These barriers can block escaping photoelectrons and lead to current balance, allowing sunlight charging to high negative levels. In a previous treatment, analytic models were limited to fast spin relative to differential charging rates so that the solutions had azimuthal symmetry around the spin axes. By introducing an associated Legendre term into the potential expansion, the azimuthal symmetry is removed, and the models can be developed to encompass any spin rate. The analysis turns up three functions of spin rate which are only known at the spin limits, but the characteristics of the charging of a rotating sphere can be explored using approximate forms which represent the basic trends. For finite spin, the sunlit side charges less (negatively) than the shade side which is in contrast to the fast spin case, where these two potentials are equal. Also, for finite spin, differential charging develops perpendicular to the sun and spin axis directions, due to the transverse motion. This transverse charging occurs at all finite spin rates, disappearing only at the zero and infinite spin limits. There is a correlated lag angle between the direction of maximum sheath radius and the sun line. Plots are given to illustrate the potential distributions representing barrier dominated sunlight charging of a spinning dielectric coated spherical satellite.
\end{abstract}

Keywords. Space plasma physics (Electrostatic structures; spacecraft sheath, wakes, charging; General or miscellaneous)

Correspondence to: S. T. Lai

(shu.lai@hanscom.af.mil)

\section{Introduction}

It is known that a satellite in sunlight can charge to substantial negative voltages, even though the photoelectron current (positive) from surfaces (Hinteregger, et al., 1959; Wrenn and Heikkila, 1973) is typically much larger than the ambient currents in space. This effect occurs because photoelectrons escaping from the satellite can be blocked by potential barriers at the surface, leading to current balance and to charging to high (negative) values. In this paper, we consider barrier dominated charging models for spinning spherical satellites. Only steady state configurations are treated, although we use a time dynamic argument to obtain time scales for the charging behavior during a spin period.

The analytic models assume Laplacian potentials exterior to the spherical satellite surface. The potentials are expanded in a series of multipoles and we consider the three lowest order terms: the monopole, dipole, and the quadrupole. The monopole-dipole model, where the satellite is non-rotating, has been treated by Besse and Rubin (1980), Mandell et al. (1978), and Higgins (1978). The monopole-quadrupole system has been treated by Tautz (2003). The monopoledipole model forms with the dipole aligned with the sun direction. In the monopole-quadrupole system, the sun line lies in the bellyband plane and the satellite is spinning fast. A combination of these models, representing charging with an arbitrary sun direction with respect to the spin pole, was developed by Tautz and Lai (2005). In that treatment approximate azimuthal symmetry was assumed, which would be the case if the satellite is rapidly rotating. Here we drop this restriction and allow the spin rate to vary from zero to the fast spin limit, which enables the models to encompass a much wider class of satellites. To simplify the analysis, we assume that the sun direction lies in the bellyband plane.

The basic equations describing the models and their parameters are given in Sect. 2. A criterion for for slow or fast spin is discussed in Sect. 3. Section 4 gives the calculation of the photoemission barrier. In Sect. 5, approximate solutions for a variable finite spin rate are outlined and in Sect. 6 plots

Published by Copernicus GmbH on behalf of the European Geosciences Union. 
are shown to describe the characteristic features of a spinning system. Section 7 contains a summary and conclusions.

\section{Description of the models}

Consider a dielectric covered spherical satellite that is spinning in sunlight. If the ambient charge density is low, which is a typical condition at geosynchronous altitudes, the potentials outside such a satellite are given approximately by a solution to Laplace's equation. In spherical coordinates, the exterior Laplacian potentials are of the form (see Magid, 1972):

$$
\begin{aligned}
& V(r, \theta, \phi)=\sum_{n} \frac{1}{r^{n+1}} \\
& \quad\left\{\sum_{m} P_{n}^{m}(x)\left[B_{n m} \cos (m \phi)+C_{n m} \sin (m \phi)\right]\right\}
\end{aligned}
$$

where $\mathrm{r}$ is the radius, $\theta$ is the polar angle and $\phi$ is the azimuth angle. The $P_{n}^{m}(x)$ are the associated Legendre polynomials (Beyer, 1973) with $\mathrm{x}=\cos (\theta)$. The sum $\Sigma_{n}$ is over $\mathrm{n}=0,1$, $2 \ldots$ and the sum $\Sigma_{m}$ is over $\mathrm{m}=0$ to $\mathrm{n}$. The constant coefficients $B_{n m}, C_{n m}$ depend on the exterior conditions.

In terms of Cartesian coordinates $(\mathrm{X}, \mathrm{Y}, \mathrm{Z})$ in the space frame of reference, the satellite is assumed to be spinning around the Z-axis with the sun direction lying along the positive $X$-axis. In this configuration, the potentials should, by symmetry, be even in $x$ (i.e. with respect to $+Z$ or $-Z$ ). We can eliminate the $B_{21}$ and $C_{21}$ terms because $P_{2}^{1}(\mathrm{x})$ is odd in $\mathrm{x}$ (the $B_{10}$ term could also be omitted at this point, but we leave it in to show the form of the equations). The $B_{22}$ term can be dropped because, when it is expressed in Cartesian coordinates, it is even in the X-coordinate and we expect to see sunlit to shade side differences for the sun on one side at $+\mathrm{X}$. The $C_{22}$ term can also be dropped for the same reason, since it is invariant under $\mathrm{X}, \mathrm{Y}$ reflections. The potential expansion for $\mathrm{n}=0,1,2$ then has the form

$$
\begin{array}{r}
V(r, \theta, \phi)=\frac{K}{r}\left\{P_{0}^{0}(x)+\frac{1}{r}\left[P_{1}^{0}(x) A_{10}+P_{1}^{1}(x)\right.\right. \\
\left.\left.\left(A_{11} \cos \phi+A_{11}^{*} \sin \phi\right)\right]+\frac{1}{r^{2}} P_{2}^{0}(x) A_{20}+\ldots\right\}
\end{array}
$$

Here the first term is the monopole, the next three are $\mathrm{Z}, \mathrm{X}$ and $\mathrm{Y}$ dipoles, and the last is the quadrupole component. All higher order terms are neglected. For convenience, in Eq. (2), we have factored out the first term so that $K=B_{00}$ represents the monopole potential and the coefficients $A_{n m}=B_{n m} / K, A_{n m} *=C_{n m} / K$ give the strength relative to the monopole. In this expression the Legendre polynomials are (Beyer, 1973 )

$$
\begin{aligned}
& P_{0}^{0}=1 ; \quad P_{1}^{0}(x)=x ; \quad P_{1}^{1}(x)=\left(1-x^{2}\right)^{1 / 2} ; \\
& P_{2}^{0}(x)=\frac{1}{2}\left(3 x^{2}-1\right)
\end{aligned}
$$

The $P_{1}^{1}$ function was absent from previous treatments because the potential was assumed to have azimuthal symmetry $(\mathrm{m}=0)$ and it is this $P_{1}^{1}$ term which enables consideration of the finite spin cases.

The $K$ parameter in the models depends on the balance of the incoming and outgoing satellite surface currents and weakly on the photoemission barrier region (photosheath). For negative charging, $K$ is less than zero. The $A_{n m}, A_{n m}{ }^{*}$ parameters, which give the strength of the non-monopole contributions to the expansion, are also used in setting current balance and they depend more sensitively on the photosheath structure. To actually determine these parameters one needs to know the environment currents, the satellite surface material properties and the photoemission model. These inputs, which can be quite complicated, are not considered in our schematic approach and we take $K$ and $A_{n m}, A_{n m}$ * to be free parameters of the models.

If we normalize to the sphere radius so that at the satellite surface, $r=1$, and substitute for the Legendre terms, Eq. (2) becomes:

$$
\begin{aligned}
V(1, \theta, \phi) & =K\left\{1+\cos \theta A_{10}+\sin \theta\left(A_{11} \cos \phi+A_{11}^{*} \sin \phi\right)\right. \\
& \left.+\frac{1}{2}\left(3 \cos ^{2} \theta-1\right) A_{20}+\ldots\right\}
\end{aligned}
$$

For a satellite spinning around the Z-axis with the sun direction towards positive $X$, the surface potentials at the coordinate nodes $+\mathrm{Z},-\mathrm{Z},+\mathrm{X},-\mathrm{X},+\mathrm{Y},-\mathrm{Y}$ can be labeled as follows:

$V_{N}=V\left(1,0^{\circ}\right.$, any $)=K\left(1+A_{10}+A_{20}\right)$

(north spin pole)

$V_{S}=V\left(1,180^{\circ}\right.$, any $)=K\left(1-A_{10}+A_{20}\right)$

(south spin pole)

$V_{F}=V\left(1,90^{\circ}, 0^{\circ}\right)=K\left(1+A_{11}-A_{20} / 2\right)$

(front, sunlit side)

$V_{B}=V\left(1,90^{\circ}, 180^{\circ}\right)=K\left(1-A_{11}-A_{20} / 2\right)$

(back, shaded side)

$V_{E}=V\left(1,90^{\circ}, 90^{\circ}\right)=K\left(1+A_{11}{ }^{*}-A_{20} / 2\right)$

(east side)

$V_{W}=V\left(1,90^{\circ},-90^{\circ}\right)=K\left(1-A_{11}^{*}-A_{20} / 2\right)$

(west side)

From the sum and difference of Eqs. (5), (6) we get

$\left(V_{S}-V_{N}\right) / 2=K\left(A_{10}\right)$

$\left(V_{S}+V_{N}\right) / 2=K\left(1+A_{20}\right)=V_{P}$

(average pole potential) 
It is apparent that if $A_{10}=0$, then $V_{S}=V_{N}$. By symmetry, this will only occur when the sun lies in the X-Y plane (bellyband). In this paper, the sun direction is assumed to lie along the $\mathrm{X}$-axis i.e. we will fix $A_{10}=0$. An approximate way to treat more general sun directions in the fast spin limit has been given in Tautz and Lai (2005).

We now take the sum and difference of Eqs. (7), (8) and Eqs. (9), (10) to get

$$
\begin{aligned}
& \left(V_{F}-V_{B}\right) / 2=K A_{11}=-D / 2 \\
& \left(V_{F}+V_{B}\right) / 2=K\left(1-A_{20} / 2\right) \\
& \left(V_{E}-V_{W}\right) / 2=K A_{11}^{*}=-d / 2 \\
& \left(V_{E}+V_{W}\right) / 2=K\left(1-A_{20} / 2\right)
\end{aligned}
$$

The variables, $D$ and $d$, denote the sun aligned and transverse differential charging respectively, and are seen to be simply related to the $\mathrm{X}$ and $\mathrm{Y}$ dipole strengths. In these equations, the signs of the potentials, including $K$, would be negative for typical charging. The potentials, normalized to $K$, would therefore be positive. For the sun direction at $+\mathrm{X}$, we expect $V_{B} / K>V_{F} / K$ and $A_{11}<0$. For satellite spin in the counter clockwise sense, we would have $V_{W} / K>V_{E} / K$, giving $A_{11}{ }^{*}<0$. Spin in the clockwise sense would correspond to $A_{11}^{*}>0$.

By comparing Eqs. (14), (16) we get

$$
V_{E}+V_{W}=V_{B}+V_{F}
$$

This condition states that, for the configuration and approximation level that we have considered, the system is diagonally balanced. We mean by this that the voltage change in any spin quadrant is the same as the change in the diagonally opposite quadrant. This can be seen in Fig. 1, which gives a top view of the satellite spin quadrants, with the sun located to the right and the rotation counter clockwise. In the figure, the symbols E, W, B, F label the east, west, back and front sides of the sphere. From Eq. (17), it can be seen that the voltage increment in the first quadrant $\left(V_{B}-V_{E}\right)$ equals the decrement in the third quadrant $\left(V_{W}-V_{F}\right)$. The same type of voltage balance holds for quadrants two and four.

Equations (14), (16) lead to

$$
\begin{aligned}
A_{20}=2\left(1-V_{M} / K\right) & =2\left(1-\left(V_{E}+V_{W}\right) / 2 K\right) \\
& =2\left(1-\left(V_{B}+V_{F}\right) / 2 K\right)
\end{aligned}
$$

where we denote by $V_{M}$ the average middle (bellyband) potential

$V_{M}=\frac{1}{4}\left(V_{E}+V_{W}+V_{F}+V_{B}\right)$

We can see that the quadrupole strength coefficient, $\mathrm{A}_{20}$, can be determined by the ratio of $V_{M}$ to $K$ and this coefficient is zero if $V_{M}=K$.

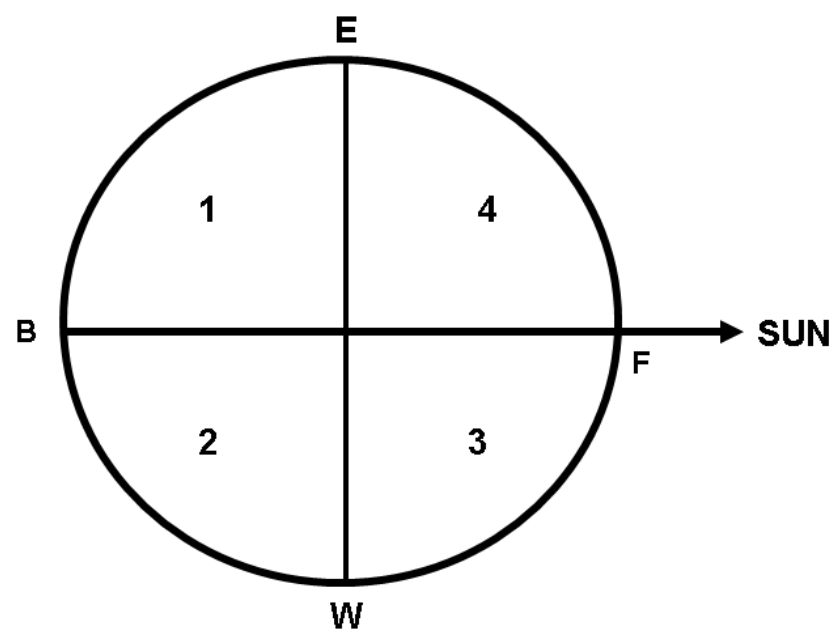

Fig. 1. A top view of the satellite spin quadrants, with the sun located to the right and the rotation counter clockwise.

The average surface potential is denoted by

$$
<V>=\frac{1}{6}\left(V_{N}+V_{S}+4 V_{M}\right)
$$

From the sum of Eqs. (5-10) we find that $\langle V\rangle=K$. This simple equation is helpful in understanding the overall charging behavior of spinning spheres. It can be written in the equivelant form

$V_{P} / K=3-2 V_{M} / K$

We see that, if the bellyband potentials change, the spin pole potentials adjust linearly. In general, any change to a surface potential will be compensated by changes to other surfaces such that the average surface potential remains at $K$.

If we are given the six node potentials, the coefficents $A_{10}$, $A_{11}, A_{11} *$ and $A_{20}$ are determined by Eqs. (11), (13), (15), (18). We now examine two limit cases for which these node potentials are known. We first look at the zero spin limit. It is known that the solution is a monopole-dipole system (Besse and Rubin, 1980) pointing in the sun direction, $X$, and given by

$$
\begin{aligned}
& V_{B}=K(1+A) \\
& V_{F}=K(1-A) \\
& V_{N}=V_{S}=V_{E}=V_{W}=K
\end{aligned}
$$

where $A>1 / 2$ is the dipole strength parameter. Solving for the coefficients, we find $A_{10}=A_{11} *=A_{20}=0$ and $A_{11}=-A$. Here $V_{M}=K$ and this makes the quadrupole term go to zero. The differential charging is given by $D=2 K A$ and $d=0$. There is also differential charging between the spin poles and bellyband which is azimuth dependent. We next look at the case 


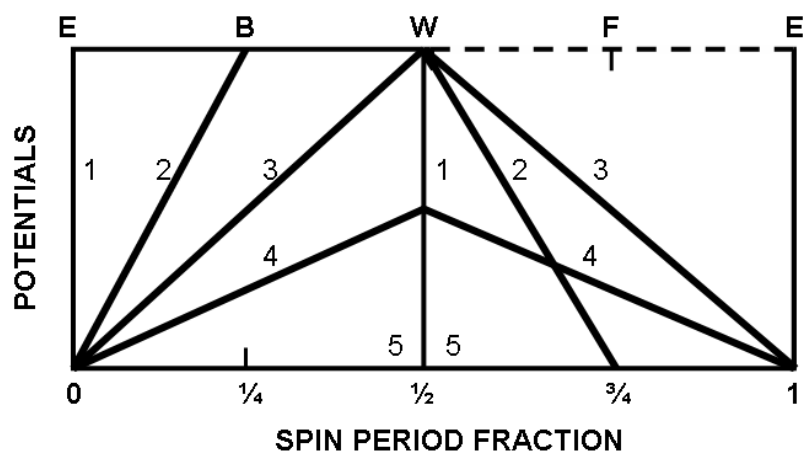

Fig. 2. Hypothetical linear charging voltages as functions of the spin period fractional times. The charging rate on the dark side is assumed to be equal to the discharge rate on the sunlit side. The horizontal dashed line represents the dark side equilibrium value. The top letters denote the east (E), back (B), west (W), and front (F) surfaces relative to the sun direction.

of infinite spin. It is known that the solution is a monopolequadrupole system (Tautz and Lai, 2005) given by

$V_{E}=V_{W}=V_{F}=V_{B}=K(1-A / 2)$

$V_{N}=V_{S}=K(1+A)$

where $A>2 / 3$ is now the quadrupole strength parameter. Again, solving for the coefficents we get $A_{10}=A_{11}=A_{11} *=0$ and $A_{20}=A$. The differential charging is here $D=d=0$ and all the bellyband potentials are equal, which characterizes the fast spin limit. There is again pole-bellyband differential charging, which in this case is azimuthally symmetric.

We have seen that, depending on the parameters $A_{10}, A_{11}$, $A_{11}$ * and $A_{20}$, we can get a zero spin or infinite spin solution. In both cases $A_{10}=0$ which signifies that the sun lies in the Xy-plane. Also, in both cases, we have $A_{11}{ }^{*}=0$, but we do not assume that this is true in general.

\section{Fast and slow spin}

We now wish to discuss the models at finite spin rates, between the limit cases of zero and infinity. To get an insight into the charging time scales, we need a means of judging whether a given spin rate is fast or slow. We can use a time dynamics argument to characterize the significant charging intervals during a rotation. The analysis takes into account that each surface element can be characterized by its capacitance per unit area and it will charge and discharge on a time scale set by that value as well as by the spin rate. There are thus two relevant times to compare: the time for equilibrium surface charging, $T$, and the satellite spin period, $\tau$. We can define a spin rate parameter, $R$, as

$R=2 T / \tau$

The factor of two is inserted because it is the spin half period which is the critical time. It should be noted that $R$ is only defined approximately. It depends on the charging time $T$, which is not precisely defined. $T$ can be estimated from the time constant for differential charging, but there is not a precise value at which a transition to the fast spin state is realized. Experimentally, the fast spin limit could be recognized by a lack of spin modulation in measured on-board surface fluxes.

Consider the behavior of an isolated surface cell on a spinning spacecraft. If the satellite is in a charging environment (Lai and Tautz, 2006) a surface on the shaded side will go negative at a rate $\mathrm{d} V / \mathrm{d} t=J / C$ where $J$ is the dark side current density and $C$ is the capacitance per unit area of the element. Typical values for an orbiting geosynchronous spacecraft are $J=1 \mu \mathrm{A} / \mathrm{m}^{2}$ and $\mathrm{C}=1 \mu \mathrm{F} / \mathrm{m}^{2}$ which gives $\mathrm{d} V / \mathrm{d} t=1$ Volt/s. If the satellite spins at one RPM, the charging on the dark side would reach 30 Volts. This is not a negligible amount for a spacecraft charging to a few hundred volts in sunlight.

To estimate time scales, we follow a cell as it moves around the spacecraft bellyband during one spin period through surface points $\mathrm{E}, \mathrm{B}, \mathrm{W}, \mathrm{F}$ and then back to $\mathrm{E}$ (see Fig. 1). The corresponding fractional times are $0,1 / 4,1 / 2$, $3 / 4$ and 1. In Fig. 2, we show five hypothetical charging curves as a function of the spin period fractional times. For simplicity, the curves are shown as linear. The charging rate on the dark side is assumed to be equal to the discharge rate on the sunlit side. The curves are drawn as diagonally balanced, in the sense discussed in Sect. 2. The horizontal dashed line represents the dark side equilibrium level, $V_{e}$. In the figure, the curves are labeled $1-5$ and locate the main regions:

1) $R=0$ - the stationary case

2) $R=1 / 2$ - the end of the very slow spin region

3) $R=1$ - the end of the slow spin region and the start of the fast spin region

4) $R=2$ - the center of the fast spin region

5) $R=\infty-$ a horizontal line, which is the fast spin limit

The parameter value $R=1$ specifies the boundary between slow and fast spin. The charging behavior is quite different between these two regions. For slow spin, the potential levels off at the dark side equilibrium level, $V_{e}$. For fast spin, the dark side potential never gets to equilbium. It is cut off at time $\tau / 2$, at a value which decreases with increasing spin.

As in Sect. 2, we denote the front to back voltage difference in the sun direction as D and the difference in the transverse direction as d. From Fig. 2, we can see that the differential charging, $\mathrm{D}$, is a maximum at zero spin and decreases with $R$. At $R=1$, the average potential on the dark side approximately equals the average on the discharge side, and $\mathrm{D}$ goes to zero, where it remains for $R>1$. The transverse differential charging, $d$, on the other hand, occurs whenever the 
satellite has finite spin. It is due to the charging up of the cell as it moves from east to west and then discharges from west to east, creating a transverse voltage difference. It only vanishes at the limits when the satellite is stationary, so that by symmetry the potentials are the same, or at the fast spin limit where all bellyband potentials are equal.

Real charging curves would be non-linear. Any curvature would smooth out the linear results. For example, hard zeros for $\mathrm{D}$ would go into small numbers. The time dynamics analysis is schematic, as the models pertain solely to the steady state and the surface is not composed of isolated cells, but it serves to identify the charging regions.

To illustrate the effect of time averaging, we calculate the potentials in the circulating cell case, which can be easily obtained for the linear curves of Fig. 2. The results are shown in Table 1. Here the time averages are taken using plus or minus one quarter of a spin period, centered on the fractional time for the potential. For this calculation, we have used potential limits $v_{e}=3 / 2$ and $v_{E}=1 / 2$, which for comparison have been chosen to give the Besse-Rubin threshold potential ratios, $\mathrm{V} / \mathrm{K}$, at zero spin (see line 1 of Table 1 ). The computed time averaged charging variables are summarised in the table. The charging behavior is similar to the dynamic cases. We note that the time averaged potentials $V_{E}, V_{W}$ are equal at $R=0$, rather than having discontinuous jumps.

We can parameterize the spin behavior, by letting the model coefficients $A_{11}, A_{11}{ }^{*}, A_{20}$, be functions of $R$. We will assume these functions have the form

$A_{n m}(R)=\alpha a_{n m}(R)$

and a similar form for $A_{11} *$. Here $\alpha$ is an overall strength parameter and the $a_{n m}(R)$ functions give the relative weights. If $\alpha$ is zero, there is no differential charging and we revert to the eclipse charging case. Using the $K, \alpha, R$ parameterization, the expression for $V$ becomes

$$
\begin{aligned}
& V(r, \theta, \phi)=\frac{K}{r} \\
& \qquad 1+\frac{\alpha}{r} \sin \theta\left[a_{11}(R) \cos \phi+a_{11}{ }^{*}(R) \sin \phi\right] \\
& \left.\quad+\frac{\alpha}{2 r^{2}}\left(3 \cos ^{2} \theta-1\right) a_{20}(R)\right\}
\end{aligned}
$$

This is the approximate potential that is used to describe a spinning sphere. The $A_{10}$ term has been set to zero. The $R$ dependence is discussed more fully in the following sections.

\section{The photoemission barrier}

For substantial negative charging to occur in sunlight it is expected that a potential barrier will form just outside the surface and trap escaping photoelectrons. Such a barrier exists if

$$
\frac{d}{d r} V(r, \theta, \phi)=0
$$

Table 1. Time averaged potentials for a rotating surface cell.

\begin{tabular}{lllllll}
\hline$R$ & $V_{B}$ & $V_{F}$ & $V_{W}$ & $V_{E}$ & $D$ & $d$ \\
\hline 0 & $3 / 2$ & $1 / 2$ & 1 & 1 & 1 & 0 \\
$1 / 4$ & $11 / 8$ & $5 / 8$ & $9 / 8$ & $7 / 8$ & $3 / 4$ & $1 / 4$ \\
$1 / 2$ & $5 / 4$ & $3 / 4$ & $5 / 4$ & $3 / 4$ & $1 / 2$ & $1 / 2$ \\
$3 / 4$ & $9 / 8$ & $7 / 8$ & $31 / 24$ & $17 / 24$ & $1 / 4$ & $7 / 12$ \\
1 & 1 & 1 & $5 / 4$ & $3 / 4$ & 0 & $1 / 2$ \\
\hline $3 / 2$ & $5 / 6$ & $5 / 6$ & 1 & $2 / 3$ & 0 & $1 / 3$ \\
2 & $3 / 4$ & $3 / 4$ & $7 / 8$ & $5 / 8$ & 0 & $1 / 4$ \\
4 & $5 / 8$ & $5 / 8$ & $11 / 16$ & $9 / 16$ & 0 & $1 / 8$ \\
$\infty$ & $1 / 2$ & $1 / 2$ & $1 / 2$ & $1 / 2$ & 0 & 0 \\
\hline
\end{tabular}

has a solution. We can write the potential, Eq. (26), in simplified form as

$$
V(r, \theta, \phi)=\frac{K}{r}\left\{1+\frac{\alpha T_{1}}{r}+\frac{\alpha T_{2}}{r^{2}}\right\}
$$

where we have defined the terms

$$
\begin{aligned}
& T_{1}=\sin \theta\left[a_{11}(R) \cos \phi+a_{11}{ }^{*}(R) \sin \phi\right] \\
& T_{2}=\frac{\left(3 \cos ^{2} \theta-1\right)}{2} a_{20}(R)
\end{aligned}
$$

We get from Eqs. (27), (28) a quadratic equation for $r$

$r^{2}+2 \alpha T_{1} r+3 \alpha T_{2}=0$

The solution $r_{b}$ for the barrier radius is thus

$r_{b}=-\alpha T_{1}+\left[\left(\alpha T_{1}\right)^{2}-3 \alpha T_{2}\right]^{1 / 2}$

Note that $r_{b}$ depends on $\alpha$ and $\theta, \phi, R$ (through $T_{1}$ and $T_{2}$ ), but not on $K$. Since the $K$ parameter does not appear in Eq. (32), it is mainly the $\alpha$ parameter which represents the photosheath structure.

We can write $r_{b}=1+s$, where $s$ is the sheath width, normalized to the sphere radius. If the sheath forms close to the sphere surface $(s \ll 1)$, we find that, neglecting quadratic terms, we have

$s=-\frac{1+2 \alpha T_{1}+3 \alpha T_{2}}{2\left(1+\alpha T_{1}\right)}$

The condition giving the threshold for charging $\left(r_{b}=1, s=0\right)$ is

$1+2 \alpha T_{1}+3 \alpha T_{2}=0$

Taking $\alpha=1$ at the threshold, we find that the values of $\mathrm{T}_{1}, \mathrm{~T}_{2}$ in the zero spin case are $T_{1}=-1 / 2, T_{2}=0$ and in the fast spin limit are $\mathrm{T}_{1}=0, \mathrm{~T}_{2}=-1 / 3$.

For a non-spinning spacecraft we expect that the maximum sheath radius would occur along the sun direction $\left(\theta=90^{\circ}, \phi=0^{\circ}\right)$. However, for a spinning satellite this is not 
necessarily true. To get the location of the maximum sheath radius, we differentiate Eq. (32) with respect to $\phi$. Since the $\phi$ dependence occurs only through $T_{1}$, we can use $\mathrm{d} T_{1} / \mathrm{d} \phi=0$, which leads to the sheath angle, $\phi_{b}$, at the maximum barrier radius, as

$\phi_{b}=\tan ^{-1}\left[a_{11}^{*}(R) / a_{11}(R)\right]$

If we evaluate the threshold condition along the line of maximum sheath radius $\left(\theta=90^{\circ}, \phi=\phi_{b}\right)$, we get from Eqs. (29), (30), (34), (35) a relation between the coefficients

$$
-a_{11}(R) \cos \phi_{b}-a_{11}^{*}(R) \sin \phi_{b}+\frac{3}{4} a_{20}(R)=\frac{1}{2}
$$

As $R$ goes from 0 to $\infty$, the coefficients will change, but they will always satisify Eq. (36) at threshold. For $\alpha>1$, the $A_{n m}(R)$ coefficients would be scaled from the $a_{n m}(R)$ values, resulting in a finite barrier radius $\left(r_{b}>1\right)$ outside the surface, with possible charging.

The model barrier height, $V_{b}$, is defined as

$V_{b}=V\left(r_{b}, \theta, \phi\right)-V(1, \theta, \phi)$

which yields

$V_{b}=\frac{K}{r_{b}}\left\{1-r_{b}+\alpha T_{1} \frac{\left(1-r_{b}^{2}\right)}{r_{b}}+\alpha T_{2} \frac{\left(1-r_{b}^{3}\right)}{r_{b}^{2}}\right\}$

In general, the ratio $V_{b} / K$ depends on $\alpha$ and $\theta, \phi, R$. At the maximum sheath radius, $T_{1}$ should be evaluated using $\phi=\phi_{b}(R)$.

At $R=0$, Eqs. (32), (37) give the characteristic equations for a monopole-dipole system (Besse and Rubin, 1980)

$r_{b}=\alpha$

$V_{b}=\frac{K}{2 \alpha}(\alpha-1)^{2}$

and as $R \rightarrow \infty$ they go into the fast spin equations (Tautz and Lai, 2005)

$r_{b}=\alpha^{1 / 2}$

$V_{b}=K\left(\frac{1}{3} \alpha+\frac{2}{3} \alpha^{-1 / 2}-1\right)$

Note that these expressions differ from ones given in former publications because the definition of $\alpha$ has changed slightly from the previously used non-monopole strength parameter. We can see directly that $\alpha=1$ gives back the threshold values $r_{b}=1, V_{b} / K=0$.

The energy of the emitted photoelectrons is low ( 1 to $2 \mathrm{eV}$, Whipple, 1981; Lai et al., 1986), and the barrier height $V_{b}$ required to stop a substantial fraction of them would only be a few volts. For high-level charging, with $K$ at hundreds of volts, the normalized barrier height $V_{b} / K$ will be near to its threshold value. It is this approximate feature of sunlight charging which allows the threshold states to be used effectively as a basis for the solutions.

The above analysis of the photosheath is based wholely on Laplace's equation. To do the photoelectron dynamics consistently would require solving Poisson's equation, with calculated electron density in the sheath region, which is beyond the scope of our schematic models. A photosheath model could be added separately and would provide a relation between the barrier height, $V_{b}$, and the sheath width s. This, combined with the current balance condition, would lead to a consistant closed system (see the comment at the end of Sect. 5). However, it can be shown that for strong differential charging, the space charge in the photosheath has only a small effect on the barrier (Mandell et al., 1978) and Laplace's equation is sufficient.

\section{Approximate solutions to the models}

In order to explore the charging behavior of a satellite rotating at finite spin, we assume approximate functional forms for the spin dependent threshold coefficents $a_{11}(R), a_{11} *(R)$, $a_{20}(R)$. The functions are estimated as follows:

1) We can get an idea as to the approximate behavior of the $a_{11}(R)$ function by examining Fig. 2 and Table 1. It is apparent that at $R=0$, (curve 1 in the figure) that the differential charging, $D$, has a maximum value. At $R=1$, (curve 3 ) the value of $D$ has dropped down to zero because the front and back potentials are equal and this condition remains for all fast spin rates (curves $\geq 3$ ). We expect the dipole response of a rotating sphere to be similar, but somewhat smoother. An approximate function that ramps down from the X-dipole solution is $D=K \exp (-\mathrm{b} R)$ and from Eq. (13)

$a_{11}(R)=-D /(2 K)=-(1 / 2) \exp (-b R)$

The factor of $b$ in the exponent is arbitrary. A larger $b$ puts the value of $D$ at $R=1$ closer to zero. In the examples below, we use $b=2.0$.

2) The behavior of the $a_{11} *(R)$ function can also be estimated from Fig. 2 and Table 1. At $R=0$, the east and west potentials are equal by symmetry (curve 1 ) and $d=0$. At $\mathrm{R}=\infty$, (curve 5) we again have $d=0$ because all bellyband potentials are equal. For intermediate spins, $d$ would go through a maximum. We first consider the region $R>1$. We can see that $d$ decreases with $R$, since it is cut off at $\tau / 2$, so that the functional form is $y_{2}(R)=\mathrm{c}(\tau /(2 T))=\mathrm{c} / R$ where $\mathrm{c}$ is a constant. For the region $R \leq 1$, the differential charging, $d$, starts from zero, ramps up, goes through a maximum somewhere before $R=1$, and then connects with the function at $R>1$. We can implement this behavior smoothly with a second degree polynomial, $y_{1}(\mathrm{R})$, satisfying: $y_{1}(0)=0, y_{1}(1)=\mathrm{y}_{2}(1)$, $d y_{1}(1) / d R=d y_{2}(1) / d R$ which yields $y_{1}=\mathrm{c} R(3-2 R)$. The coefficient at $R \leq 1$ is then determined using $y_{1}$ in Eq. (15)

$\left.a_{11}^{*}(R)=-d /(2 K)=-c^{\prime} R(3-2 R) 2\right)$ 
and for $R \geq 1$, using $y_{2}$ it is

$a_{11}^{*}(R)=-c^{\prime} / R$

where $c^{\prime}=c /(2 K)$. A value for $c^{\prime}$ can be estimated by assuming typical physical conditions at $R=1$. As described in Sect. 3 , if we have a satellite spinning at 1 RPM there could be about 30 Volts of transverse charging and, given a representative monopole charging level of 500 Volts, we find $c^{\prime}=30 / 1000=0.03$.

3) Rather than introducing an independent estimate for the $a_{20}(R)$ function, we set the quadrupole coefficient by a physical condition. For a sheath barrier to occur exterior to the sphere surface, the system must be above threshold, specified by condition Eq. (36), which gives

$a_{20}(R)=\frac{4}{3}\left[\frac{1}{2}+a_{11}(R) \cos \phi_{b}+a_{11}{ }^{*}(R) \sin \phi_{b}\right]$

This expression has reasonable limits since, at $R=0$ the quadrupole term is equal to zero (see Eq. 22) and, at $R=\infty$ it goes into $2 / 3$, the fast spin threshold value (as in Eq. 23).

The above functions enable us to determine potential coefficients for a spinning sphere at the charging threshold. To get the actual solution potentials one has to scale these coefficients by $\alpha$. The functions represent smoothly the trends and limits. The real functions are unknown, but these approximate forms are sufficient to illustrate the main features.

In setting up these functions two new "free" parameters $b$ and $c^{\prime}$ have been introduced. These parameters are not really free, since in a real physical charging case they would be determined from material and plasma properties. They are similar to $K$ and $\alpha$ in that they would be known if the problem was completely specified. For example, $K$ and $\alpha$ could be calculated consistently as follows. The existance of a photosheath model would provide a relation $B(\mathrm{~s})$ where $B$ is the photoemission barrier height. The multipole models provide the scaling function $V_{b} / K=f(\mathrm{~s})$. Taking the ratio of these two quantities would yield $K(\mathrm{~s})=K(\alpha)$. The $\alpha$ variable could be varied to determine $\mathrm{K}$ and thereby obtain the surface potentials. Then, using a current collection model, the plasma incident fluxes at the surface could be calculated. Interactions of the incident particles with the surface, using a materials model, would yield the net surface currents. The value of $\alpha$ could be moved until current balance at the satellite surface was obtained and this would give a consistant solution.

\section{Characteristics of the models}

To illustrate the $R$ dependence of the models, we show representative plots. The various limiting values for the plot variables, at $R=0$ and $R=\infty$, are summarized in Table 2. All potentials shown are normalized to $K$ and are positive. In a real physical case, the unnormalized potentials and $K$ would be typically negative.
Table 2. The model threshold parameters at the spin limits.

\begin{tabular}{lll}
\hline & $R=0$ & $R=\infty$ \\
\hline$a_{11}(R)$ & $-1 / 2$ & 0 \\
$a_{11} *(R)$ & 0 & 0 \\
$a_{20}(R)$ & 0 & $2 / 3$ \\
$V_{M} / K$ & 1 & $2 / 3$ \\
$V_{P} / K$ & 1 & $5 / 3$ \\
$\phi_{b}$ & 0 & 90 \\
$r_{b}$ & $\alpha$ & $\alpha 1 / 2$ \\
$V_{b} / K$ & $(\alpha-1)^{2} /(2 \alpha)$ & $\left(1 / 3 \alpha+2 / 3 \alpha^{-1 / 2}-1\right)$ \\
\hline
\end{tabular}

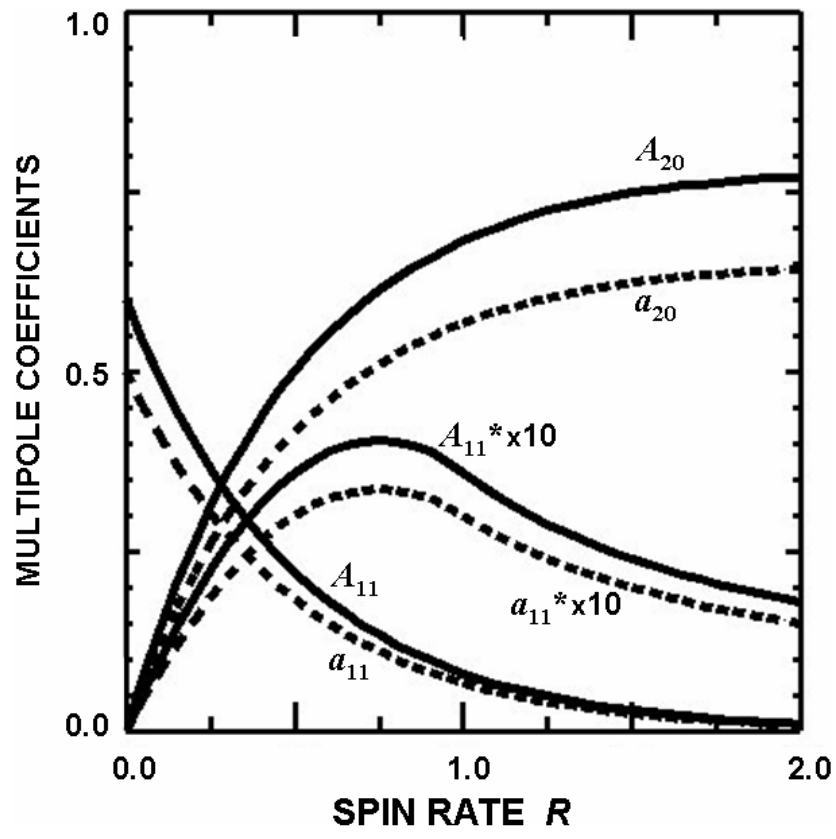

Fig. 3. Representative plots showing the $R$ dependence of the absolute value of the multipole coefficients. The solid lines are for $\alpha=1.2$ and the dashed lines for alpha $=1.0$. The $A_{11}$ terms are multiplied by 10 for clarity. The differential charging voltages, $D$ and $d$, are obtained by multiplying $A_{11}$ and $A_{11}^{*}$ by $-2 K$.

In Fig. 3 we show the assumed $R$ dependence of the coefficients $-A_{11},-A_{11}{ }^{*}$ ( $\times 10$ for clarity), $A_{20}$. The curves are given for two values of $\alpha$, corresponding to threshold $(\alpha=1)$ and to a finite sheath radius $(\alpha=1.2)$. The functions $A_{11}$ and $A_{11}{ }^{*}$ are the drivers of the models ( $A_{20}$ is set from them). The amount of differential charging, $D$ and $d$, can be scaled from the coefficients $A_{11}$ and $A_{11}^{*}$, by multiplying by $-2 K$. The threshold curves are indicated by a dashed line. The term $-a_{11}$ ramps down from $1 / 2$ to 0 . The term $-a_{11}{ }^{*}$ ramps up from 0 , goes through a maximum before $R=1$, then relaxes back to 0 at $R=\infty$. The term $a_{20}$ ramps up from 0 and goes to $2 / 3$ at $R=\infty$.

In Fig. 4, we show the average bellyband and spin pole potentials at $\alpha=1.0,1.2$. The threshold curves are indicated 


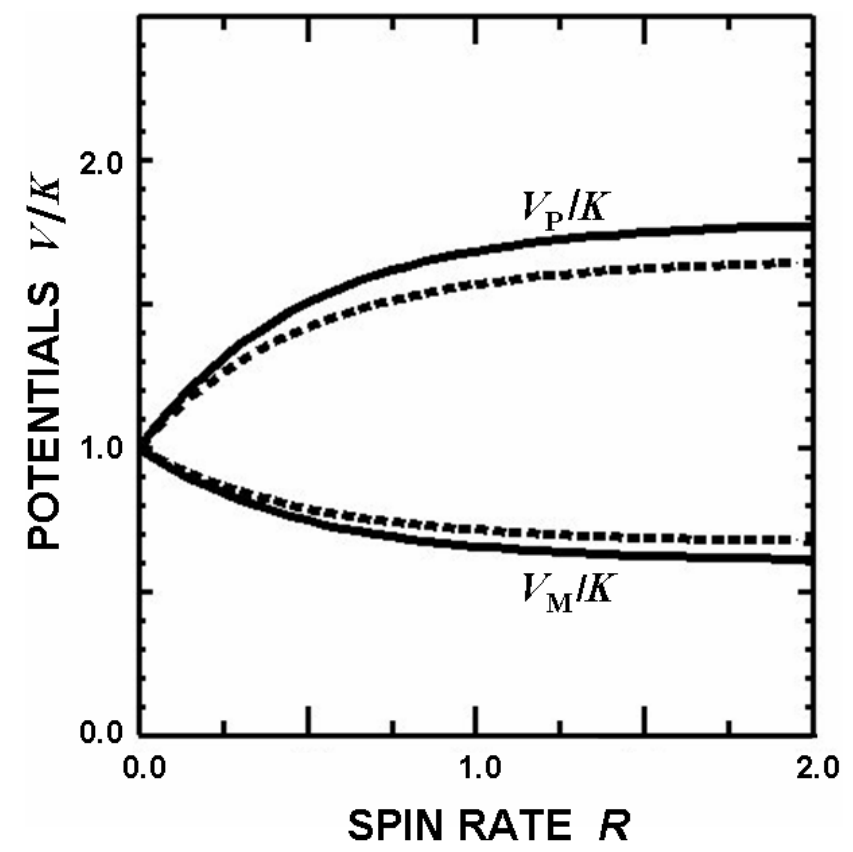

Fig. 4. The average normalized bellyband potential, $V_{M} / K$, and the average spin pole potential, $V_{P} / K$, versus $R$. The dashed lines indicate the threshold values.

by dashed lines. This plot shows how the potentials adjust to changing spin rates. As the satellite spins faster, the quadrupole term increases from zero so that $V_{M} / K$ goes lower via (from Eq. 18)

$\frac{V_{M}}{K}=1-\frac{A_{20}(R)}{2}$

According to Eq. (21), the average spin pole potential, $V_{P}$, must rise so that the average surface voltage remains at $K$. This behavior of $V_{M} / K$ and $V_{P} / K$ is plotted in Fig. 4 and shows that the average pole-bellyband differential charging increases with the spin rate. Since the sun aligned and transverse differential charging fall off with increasing spin, the pole-bellyband charging is the dominant differential voltage in the fast spin limit. The normalized difference between $V_{P}$ and $V_{M}$, using Eq. (21), is

$$
\frac{\left(V_{P}-V_{M}\right)}{K}=3\left(1-\frac{V_{M}}{K}\right)
$$

At $R=0$, the difference is 0 , corresponding the $\mathrm{X}$-dipole solution, while at $R=\infty$ it goes into the fast spin threshold value of 1 . This indicates that the potentials have changed from a monopole-dipole configuration to the "dumbell" shape of the monopole-quadrupole model. For intermediate spin rates, the potential is formed from the monopole term, a combination of an X-dipole and Y-dipole which gives differential charging in the bellyband plane, and the quadrupole component which is axially symmetric. As $R$ increases, the quadrupole term becomes stronger and brings in azimuthal smoothing to the solutions.

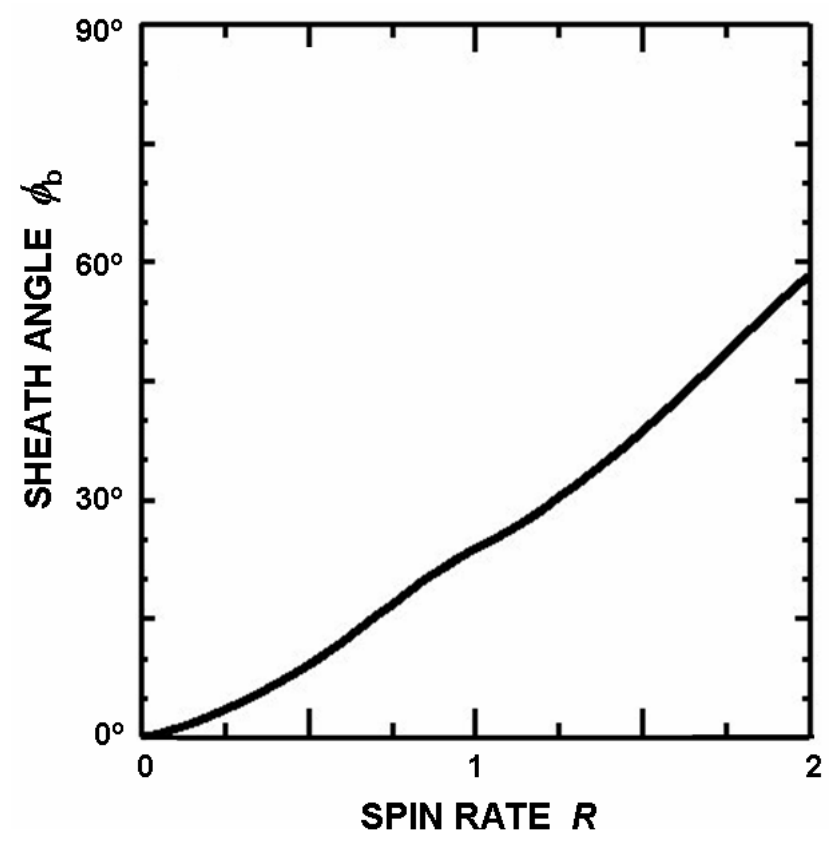

Fig. 5. The photoemission sheath angle, $\phi_{b}$, at the maximum sheath radius, versus $R$.

In Fig. 5, we show the sheath angle, $\phi_{b}$, at $\alpha=1.0$ (there is no variation with $\alpha$ ). The sheath angle increases monotonically from zero at $R=0$. At the fast spin limit it goes to $90^{\circ}$. The slight wiggle in the curve is caused by $a_{11}$ * going through a maximum near $R=1$.

Figures 6 and 7 show the sheath radius and sheath height at $\alpha=1.1,1.2$ and 1.3. In both of these figures, the threshold corresponds to the abscissa of the plots. The figures indicate that, at constant $\alpha$, the sheath becomes tighter and the barrier height lessens as $R$ increases. This effect is most apparent at $R<1$.

Figures 8 to 10 show the model solutions, for three spin rates, in the physical space surrounding the sphere. The contour plots show a $Y=0$ slice of data expressed in $\mathrm{X}, \mathrm{Y}, \mathrm{Z}$ coordinates, which are normalized to the sphere radius. In these figures, the orientation symbols B, F would be to the left and right and the $\mathrm{E}, \mathrm{W}$ symbols would be out of the page. The $\alpha$ parameter is 1.2. The sun direction, as indicated by a white line, is to the right. The plots are given for $R=0,1 / 2,1$. In the figures, one can see the progression from an X-dipole dominated, zero spin case to the quadrupole dominated solution at fast spin. The most interesting case is depicted in Fig. 9, which shows the solution at intermediate spin. It is evident that there is a diminishment of the shaded side potentials relative to the $R=0$ solution, due to the effect of azimuthal smoothing from the quadrupole term.

Figure 11 depicts the $R=1 / 2$ solution from above $(Z=0$ plane). In this plot, the orientation symbols $\mathrm{E}, \mathrm{W}, \mathrm{B}, \mathrm{F}$ would be at the same locations as in Fig. 1. Since we are 


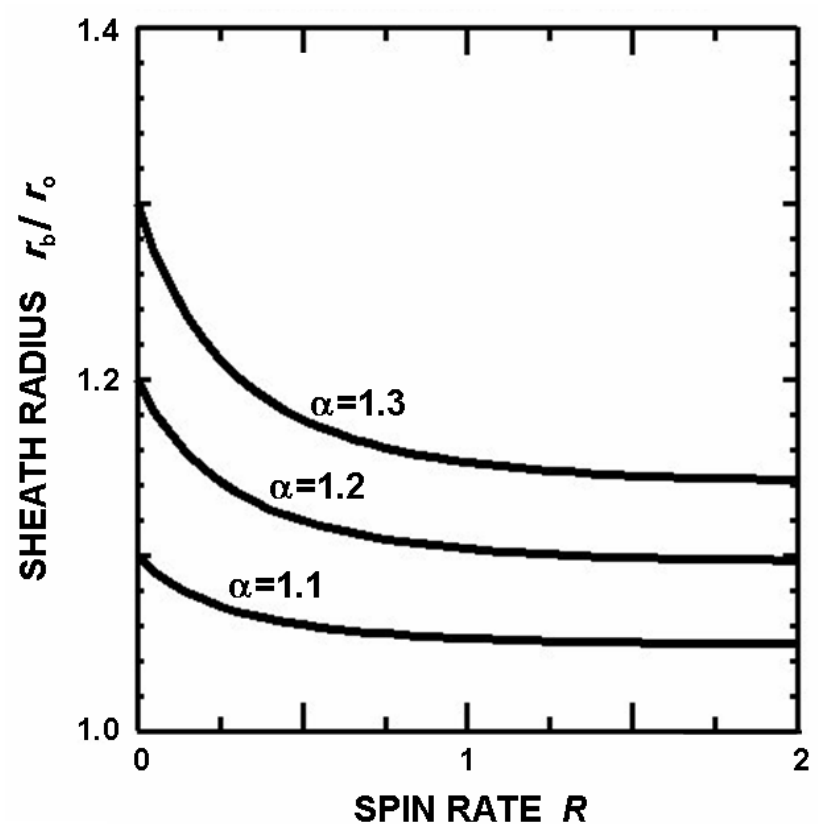

Fig. 6. The photoemission sheath radius, $r_{b}$, at $\phi_{b}$, versus $R$.

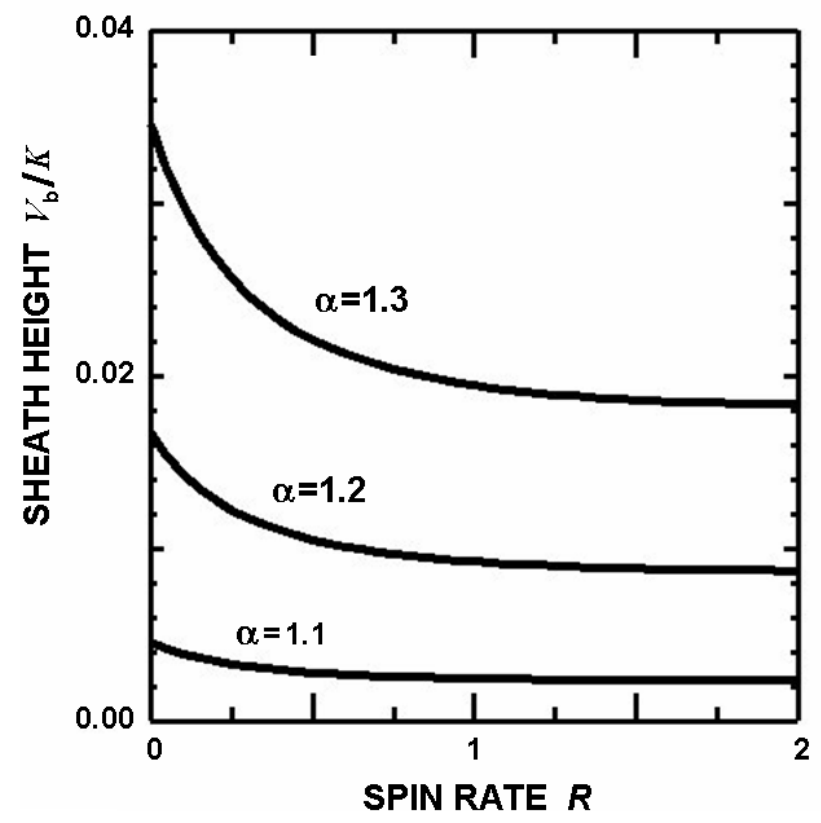

Fig. 7. The normalized photoemission sheath barrier height, $V_{b} / K$, at $\phi_{b}$, versus $R$.

at intermediate spin, there is strong azimuthal dependence, with relatively large front to back differential charging. The transverse differential charging is made evident by the deviation from $+\mathrm{Y},-\mathrm{Y}$ symmetry. For this case, the sheath angle is $\phi_{b}=9.3$ degrees, which causes the shift in the potentials, clearly seen on the forward side of the sphere. The satellite

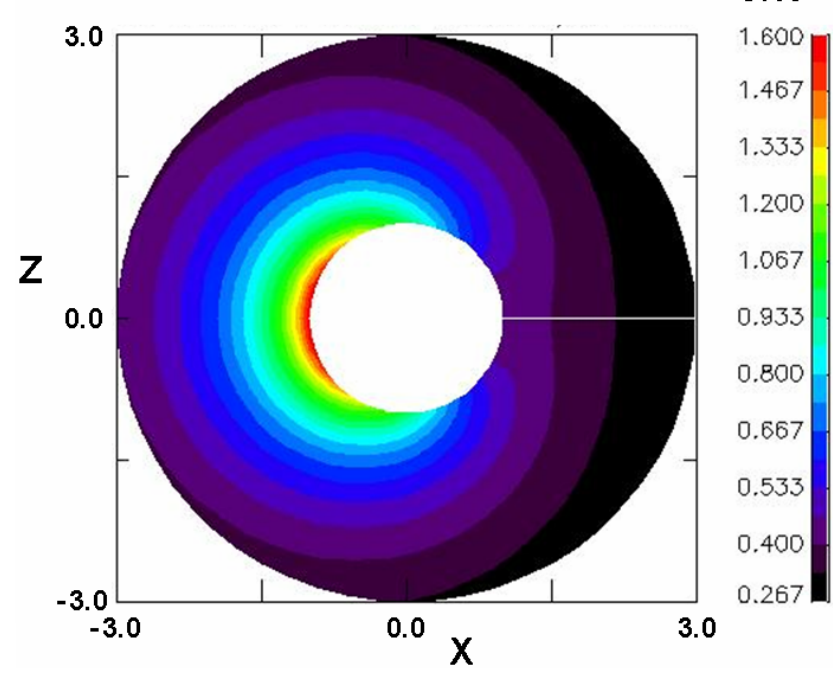

Fig. 8. Normalized potentials for the case $R=0.0, \alpha=1.2, \phi_{b}=0$, $\mathrm{Y}=0$.

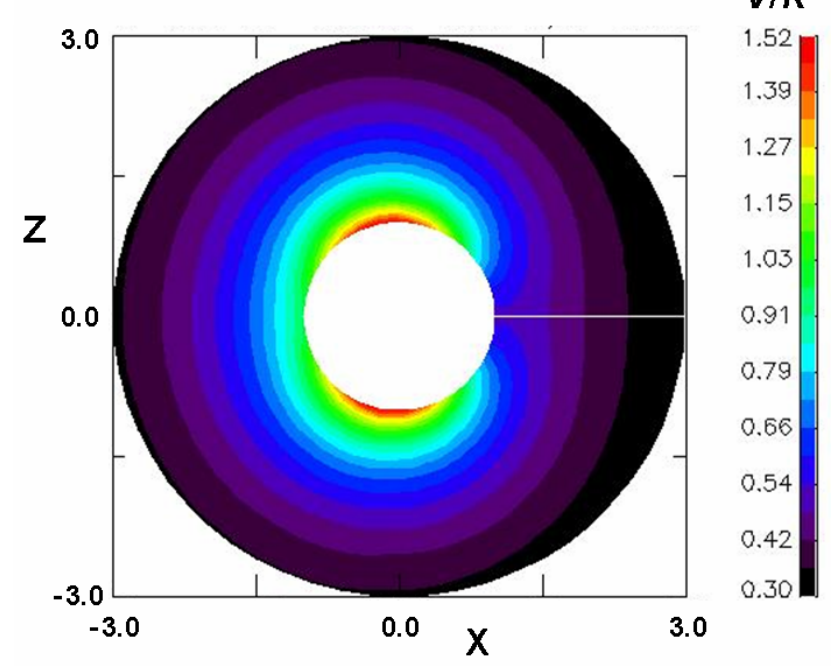

Fig. 9. Normalized potentials for the case $R=0.5, \alpha=1.2, \phi_{b}=9.3$, $\mathrm{Y}=0$.

spin is here assumed to be counter-clockwise, but an equivalent view would be that of a stationary satellite, with the sun rotating around it in the clockwise sense. Looked at this way, a positive $\phi_{b}$ represents a lag angle with respect to the moving sun line.

We remark on some limitations of the models with regard to currents. In a real charging case, satellite surface currents can be quite complex. In the models, current balance is assumed but the spatial distribution of the surface currents is not detailed. During charging, it is expected that a net negative current enters at the shaded surfaces (mainly electrons, with photoemission absent) and a net positive 


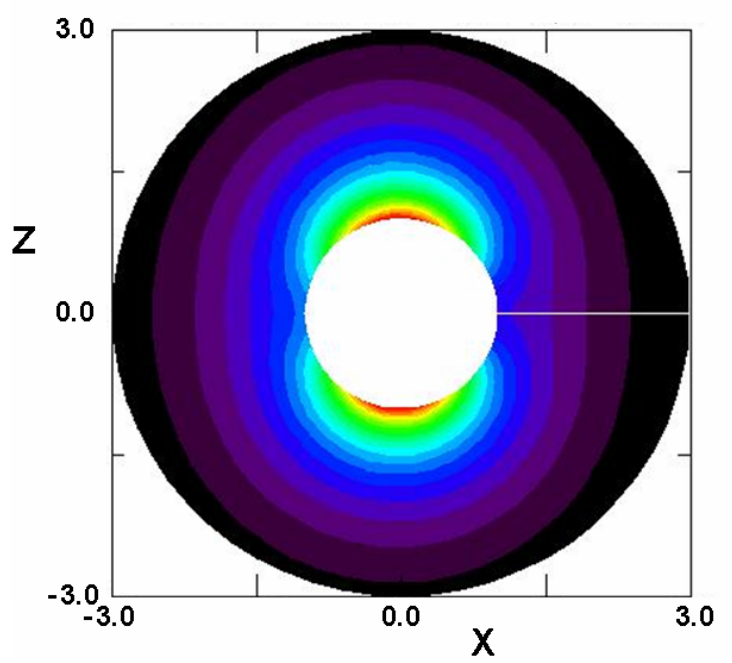

VIK

1.68

1.54

1.41

1.27

1.13

1.00

0.86

0.72

0.59

0.45

0.31

Fig. 10. Normalized potentials for the case $R=1.0, \alpha=1.2, \phi_{b}=23.9$, $\mathrm{Y}=0$.

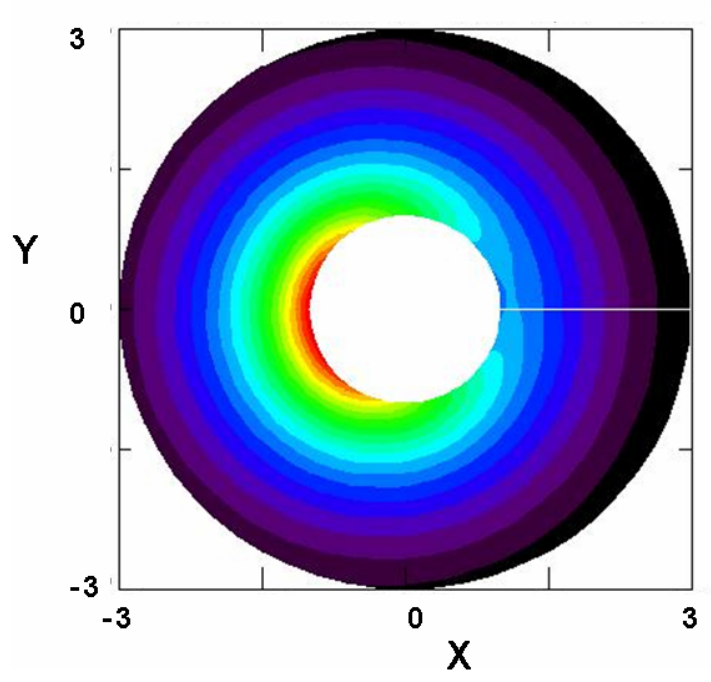

VIK

0.97

0.91

0.84

0.77

0.70

0.64

0.57

0.50

0.43

0.37

0.30

Fig. 11. Normalized potentials for the case $R=0.5, \alpha=1.2, \phi_{b}=9.3$, $\mathrm{Z}=0$.

current (mainly the unsuppressed high energy tail of the photoemission spectrum) occurs at the sunlit areas. The net current at a particular surface element would depend on the material interactions with the incident fluxes and these are not treated by the models. Another model limitation is that charge transport through the satellite by means of electrical connections is not considered. Such conduction currents would tend to reduce the degree of differential charging.

In the present models, barrier formation and nonmonotonicity of potentials are due to constraints imposed by the Legendre potential solution considered. The plots 3 to 11 above represent charging configurations which are based on typical spacecraft conditions. The models will give a better representation of real physical charging as the spaceraft/plasma conditions approach those of the theory. In order to verify the theory, it is necessary to have measurements of potential distributions on a spinning satellite with similar conditions of the models. We are not aware of such measurements. A suite of computer simulations of sunlight charging, done over a wide range of spins and spacecraft/plasma conditions, could be carried out to verify the theory and to better establish the scope and limitations of the models.

\section{Summary and conclusions}

We have generalized previous analytic models for daylight charging of a fast spinning, spherical, dielectric-covered spacecraft to treat finite rotation rates, for the case where the sun direction lies in the satellite bellyband plane. The finite spin rate is implemented by including an associated Legendre term in the potential expansion. This term removes the axial symmetry from the models and allows differential charging between back (shaded) to front (sunlit) and also between transverse surfaces during a rotation. The solution is developed by introducing a rotation parameter which spans the spin rates from the zero spin case to the fast spin limit. Plots of the solutions with varying spin show that the rotation rate is an important parameter in determining the potential distribution.

The models are limited to daylight equilibrium charging with the sun direction in the spin plane. It is assumed that the ambient density is low enough that Laplace's equation for potentials is valid. Multipole terms higher than the quadrupole are not considered in the potential expansion. Self consistant photoemission dynamics is not addressed and there is a presumption of high-level charging. The satellite body is assumed to be spherical and covered with dielectric film of uniform resistivity. The spacecraft surfaces are not coupled via electrical connections. Surface material properties and current collection algorithms have not been considered. These many assumptions are sufficient to allow a solution for barrier dominated sunlight charging to be described analytically. Based on the models, the following (not all independent) general remarks may be made:

- The basic time scales for charging are obtained by comparing the the dark side equilibrium surface charging time and the satellite spin half period.

_ "Slow spin" occurs when the dark side surface charging time is short with respect to the spin half period and "fast spin" corresponds to a charging time greater than the half period.

- In the potential expansion, the monopole term is always present. The X-dipole term is largest at zero spin and ramps down. The Y-dipole term ramps up to a maximum, then relaxes back down and it occurs at all spin 
rates except zero and infinity. The quadrupole component is zero at zero spin and is the dominant term at fast spin.

- As the satellite spins up, the quadrupole term becomes stronger and brings in azimuthal smoothing until, at fast spin, the bellyband potentials are all equal.

- The photoemission barrier height need only be a few volts to suppress photoelectrons, so that for high-level charging the solutions are close to the threshold configurations. The barrier radius and height tend to decrease with increasing spin.

- The average surface potential is equal to the monopole value. Changes to the bellyband potentials are linearly correlated with changes in the potentials at the spin poles.

- The differential voltage along the sun line ramps down from a maximum for a stationary satellite and goes towards zero when the surface charging time approaches one half the period of a spinning spacecraft.

- A differential voltage transverse to the sun line and spin axis develops whenever there is a departure from the zero spin or infinite spin limit and there is a correlated lag angle between the direction of the maximum sheath barrier and the sun line.

- There is a differential voltage (azimuth dependent) between the spin poles and the bellyband surfaces. In the fast spin limit, it becomes azimuthally symmetric and represents the dominant differential charging.

- At the zero spin limit the monople-dipole system is recovered and at the infinite spin limit the monopolequadrupole system occurs. At finite spin there is a combination of the monopole, $\mathrm{X}$ and $\mathrm{Y}$ dipole, and quadrupole terms.

These are the characteristic features describing charging of a uniform non-conducting satellite spinning around the Zaxis at a finite rate with the sun direction along the $\mathrm{X}$-axis. The models describe schematically the shape of the average potential distribution surrounding a charged spherical spacecraft in a low density space plasma and could serve as a reference point for sunlight charging of a spinning satellite.

\section{Appendix A}

\section{Nomenclature}

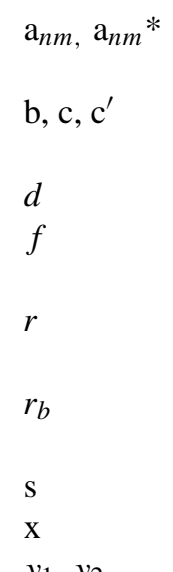

$y_{1}, y_{2}$

$A_{n m}, B_{n m}, C_{n m}$

$A_{11}, A_{11}^{*}, A_{20}$

$D$

K

$P_{n}^{m}$

$R$

$\mathrm{T}$

$\mathrm{T}_{1}, \mathrm{~T}_{2}$

$\mathrm{X}, \mathrm{Y}, \mathrm{Z}$

V

$V_{b}$

$V_{E}, V_{W}, V_{B}, V_{F}$

$V_{N}, V_{S}$

$V_{M}$

$V_{P}$

$\alpha$

$\theta$

$\phi$

$\phi_{b}$

$\tau$

$v_{e}, v_{E}$ relative coefficients in Legendre expansion

model differential charging param-

eters

transverse differential potential

scaling function of barrier height

over monopole strength

radial distance from the center of the sphere

photoemission barrier radius from center of the sphere

photoemission sheath width

polar angle function $\mathrm{x}=\cos \theta$

function terms in the $\mathrm{a}_{11} *$ coeffi-

cient

general coefficients of Legendre expansion

spin rate dependent Legendre coefficients

sun aligned differential potential

monopole potential strength param-

eter

associated Legendre polynomial

dimensionless spin rate parameter

time for equlibrium surface charging

terms in the coefficients of Legendre expansion

Cartesian coordinates in the space frame of reference

surface potential

photoemission barrier potential

surface potentials at the belly band

(east,west, back, and front sides)

surface potentials at the north and south spin poles

average potential at the middle (bellyband)

average spin pole potential

non-monopole strength parameter

polar angle

azimuth angle

photoemission sheath angle

satellite spin period

potential limits for time averages calculation
Acknowledgements. We wish to thank the referees for valuable comments. The work of M. Tautz was done under AFRL contract F19628-00-C-0089. The website of AER, Inc. is at http: //www.aer.com. 
Topical Editor I. A. Daglis thanks three referees for their help in evaluating this paper.

\section{References}

Besse, A. and Rubin, A.: A simple analysis of spacecraft charging involving blocked photoelectron currents, J. Geophys. Res., 85(A5), 2324-2328, 1980.

Beyer, W. H. (Ed.): Standard Mathematical Tables and Formulae, CRC Press, Boston, 1973.

Feuenbacher, B. and Fitton, B.: Experimental investigation of photoemission from satellite surface materials, J. Appl. Phys., 43, $1563,1972$.

Hinteregger, H. E., Damon, K. R., and Hall, L. A.: Analysis of photoelectrons from solar extreme ultraviolet, J. Geophys. Res., 64, 961-964, 1959.

Higgins, D.: An analytic model of multi-dimensional spacecraft charging fields and potentials, IEEE Trans. Nuc. Sci., 26(6), 5162-5167, 1979.

Lai, S. T., Cohen, H. A., Aggson, T. L., and McNeil, W. J.: Charging of booms on a satellite rotating in sunlight, J. Geophys. Res., 91(A11), 12 137-12 141, 1986.
Lai, S. T. and Tautz, M.: High-level spacecraft charging in eclipse at geosynchronous altitudes: A statistical study, J. Geophys. Res., 111, A09201, doi:10.1029/2004JA010733, 2006.

Magid, L. M.: Electromagnetic Fields, Energy, and Waves, Wiley Publishers, NY, 1972.

Mandell, M., Katz, I., Schnuelle, G., Steen, P., and Roche, I: The decrease in effective photo- currents due to saddle points in electrostatic potentials near differentially charged spacecraft, IEEE Trans. Nuc. Sci., 26(6), 1313, 1978.

Tautz, M.: Analytic models for sunlight charging of a rapidly spinning satellite, AFGL-TR-2003-1557, ADA416912, 2003.

Tautz, M. and Lai, S. T.: Analytical models for a rapidly spinning spherical satellite charging in sunlight, J. Geophys. Res., 110, A07220, doi:10.1029/2004JA010787, 2005.

Whipple, E. C.: Potentials of surfaces in space, Reports on Progress in Physics, 44, 1197-1250, 1981.

Wrenn, G. L. and Heikkila, W. L.: Photoelectrons emitted from ISIS spacecraft, in: Photons and Particle Interactions with Surfaces in Spaces, edited by: Grard, R. J. L., D. Reidel publishers, Hingham, Mass, 1973. 